\title{
Rete Ovarii Cystadenoma
}

National Cancer Institute

\section{Source}

National Cancer Institute. Rete Ovarii Cystadenoma. NCI Thesaurus. Code C40019.

An exceptionally rare cystadenoma that arises from the rete ovarii. 